\title{
Acute bronchiolitis: Why put an IV line?
}

\author{
Sébastien Redant ${ }^{1,2}$,Nora Nehar-Stern ${ }^{1}$, Patrick M. Honoré ${ }^{2}$, Rachid Attou ${ }^{2}$, \\ Caroline Haggenmacher ${ }^{1}$, Ashita Tolwani ${ }^{3}$, David De Bels ${ }^{2}$, Dominique Biarent ${ }^{1}$ \\ 'Emergency Department, Hôpital Universitaire des Enfants Reine Fabiola (HUDERF), UniversitéLibre de \\ Bruxelles (ULB), Belgium; \\ 2ICU Department, Centre Hospitalier Universitaire Brugmann, Université Libre de Bruxelles (ULB), Belgium; \\ ${ }^{3}$ Division of Nephrology, University of Alabama at Birmingham School of Medicine, Birmingham, AL, USA
}

\section{ABSTRACT}

Background: Acute bronchiolitis is the most frequent cause of respiratory distress in pediatric emergency medicine. The risk of respiratory failure is frequently over evaluated, and results in systematic vascular access. Methods: We conducted a prospective observational study in children under 18 months of age hospitalized for bronchiolitis. The aim of the study was to evaluate whether catheter insertion was useful for management. We monitored the number of catheters inserted in the emergency department and their subsequent use for rapid sequence intubation, adrenaline administration, or antimicrobial therapy. We recorded the number of secondary pediatric intensive care unit (ICU) admissions. Results: We followed 162 patients and compared two populations, children with (population $\mathrm{A}, n=35$ ) and without (population $\mathrm{B}$ $n=127$ ) catheter insertion. There were no significant differences in age, oxygen saturation, heart rate, c-reactive protein, neutrophil count and the number of times nebulization was conducted at admission. Population $\mathrm{A}$ compared to $\mathrm{B}$ had a significantly higher temperature ( $38.1 \pm 0.9$ vs. $\left.37.6 \pm 0.7^{\circ} \mathrm{C}, P=0.004\right)$ and respiratory rate $(64 \pm 13$ vs. $59 \pm 17, P=0.033)$. Twelve patients were secondarily transferred to pediatric ICU, 3 from population $A$ and 9 from $B$ (NS). In a multivariate analysis, no significant relationship was found between ICU admission, venous access placement and potential confounding factors (pneumonia, age $<6$ months, age $<3$ months, food intake $<60 \%$, temperature $>38^{\circ} \mathrm{C}$, heart rate $>180 \mathrm{bpm}$, respiratory rate $>60 / \mathrm{min}, \mathrm{SpO}_{2}<95 \%, \mathrm{Spo}_{2}<90 \%$, oxygen therapy, positive respiratory syncytial virus [RSV] sampling). Except for antimicrobial therapy $(n=32)$, catheters inserted in the emergency department were used in 5 patients for intravenous rehydration and in one patient in pediatric ICU for rapid sequence intubation. Conclusions: There were no life-threatening events that required immediate venous access for cardiopulmonary resuscitation. Medical treatment could be administered orally or via nasogastric tube in most cases. Peripheral catheterization was useless in immediate emergency management and only one child required a differed rapid sequence intubation.

Key words: bronchiolitis, catheter, hydration, resuscitation

\section{INTRODUCTION}

Bronchiolitis is one of the main reasons of consultations in pediatric emergency department (ED). In 2006, the American Academy of Pediatrics (AAP) recommended the initiation of intravenous (IV) hydration in patients with bronchiolitis when oral intake do not cover at least $50 \%$ of the needs. ${ }^{[1]}$ The IV route was then favored with the theoretical advantage of improving respiratory function and reducing the risk of broncho inhalation. No studies have validated this practice. ${ }^{[2]}$ The main disadvantage of IV hydration is to induce a catabolic state by decreasing caloric intake in the presence of increased metabolic demand linked to infection and respiratory stress. ${ }^{[2]}$ Similarly, infusion fluids are sometimes hypotonic and may induce hyponatremia, particularly in the presence of inappropriate antidiuretic hormone secretion syndrome (SIADH). ${ }^{[3]}$ Two randomized studies have shown that there was no advantage of the 
IV route compared to the nasogastric tube (NGT) in terms of duration of oxygen therapy, length of stay and recovery time of autonomy. ${ }^{[4]}$ Thus, AAP warned against IV rehydration risk and recommended hydration by oral or NGT. ${ }^{[3]}$

Differences in practice are found in the literature. Macias et al. observed in a multicenter trial that intravenous placement ranged from $38 \%$ to $93 \%$ of patients per center. ${ }^{[4]}$ Similarly, in the study on variability in inpatient management of children hospitalized with bronchiolitis, 9 centers had guidelines concerning the management of fluid administration in bronchiolitis. Seven centers recommend intra-venous rehydration exclusively in the acute phase, NGT being reserved for the recovery phase. Five centers describe NGT as an alternative when IV route is unavailable. Two centers recommend nasogastric tube for moderate bronchiolitis but leave the choice between the NGT and the IV route in severe cases. ${ }^{[5]}$

Differences of practice existed within our institution with respect to venous catheter insertion. Some of our hospital departments asked for systematic venous access (VA) to anticipate a secondary complication by complete intravenous hydration or in case of an emergency. Systematic VA is often difficult, time and personnel consuming, and induces stress and pain to the child. The proportion of these catheters used for rehydration or intravenous drug therapy in advanced resuscitation for a rapid sequence intubation (RSI) or adrenaline injection is not known. The aim of the study was to evaluate whether catheter placement in acute bronchiolitis presenting in the emergency department and being hospitalized was useful in helping the management of early or late respiratory failure.

\section{PATIENTS AND METHODS}

We conducted a prospective observational study in the emergency department of the Hôpital Universitaire Des Enfants Reine Fabiola (HUDERF) after hospital ethic committee approval (CEH n ${ }^{\circ}$ 63/14), all the children aged 0-18 months with oxygen-requiring bronchiolitis and/or loss of food autonomy who were hospitalized in the general wards (excluding pediatric intensive care unit [PICU] and continuing care) were consecutively enrolled in the study. Patients requiring ventilatory or circulatory support (noninvasive [NIV] or invasive ventilation, extra-corporeal membrane oxygenation (ECMO), patients with peripheral vascular access (PVA) placed in another institution, patients transferred to another hospital or directly admitted to the PICU were excluded from the study.

The primary objective was to evaluate the number of patients in whom a management requiring a VA was needed.

The variables collected were age, weight, vital parameters (heart rate, respiratory, blood pressure and oxygen saturation), c-reactive protein (CRP), Neutrophil count and feeding quantity. When inserting a vascular catheter, the number of catheter insertion trials, number of sites, use of eutectic mixture of $25 \mathrm{mg} / \mathrm{g}$ lignocaine plus 25 $\mathrm{mg} / \mathrm{g}$ prilocaine anesthesia (EMLA), use of intravenous antibiotics, intravenous corticosteroids, prescription of IV fluids, resuscitation, rapid sequence intubation and cardiopulmonary resuscitation were collected.

Data on the insertion of the vascular catheter in the emergency room (ER) were collected prospectively. The length of the follow-up was limited to the duration of the hospital stay. These data were collected and encoded on an Access database and verified by double reading. The statistics were performed using the IBM SPSS 23 software with a significance level of $P<0.05$. A KolmogorovSmirnov test allowed us to evaluate the parametric and non-parametric data. For the parametric data, we used a Student's $t$-test to compare the 2 subpopulations. For non-parametric data, a Mann-Whitney test was used. For categorical date, we used a Fisher's exact test. We compared populations with and without VA inserted in the ED as well as the patients admitted or not to ICU.

\section{RESULTS}

From October 1, 2014 till March 31, 2015, 192 patients were hospitalized for acute bronchiolitis. Thirty patients were excluded ( 20 were transferred from another hospital, 3 were admitted directly to PICU, and 7 were carriers of chronic pathologies). 162 consecutive patients were included, of whom $35(22 \%)$ patients had vascular access inserted in the ED (Table 1).

The comparison of the population (pop) with PVA placement (pop A, $n=35$ ) and without PVA placement in the ED (pop B, $n=127$ ), showed no significant difference in baseline characteristics (age, oxygen saturation, heart rate, CRP and neutrophil counts, and total number of aerosols in emergency rooms [Table 2]). Pop A compared to B had a significantly higher temperature $(38.1 \pm 0.9$ vs. $37.6 \pm$ $0.7, P=0.004)$, and a significantly higher respiratory rate (64 \pm 13 vs. $59 \pm 17, P=0.033$ ). We had no deaths in our series. The emergency procedure required an average of $2 \pm$ 1.57 trials on $1.76 \pm 1.32$ different sites. EMLA cream was used in 35\% of cases. Four patients were given an infusion. Twelve patients received antibiotics in the emergency ward, 11 for radiological infiltrate, one patient under one month for temperature (Figure 1). All bacterial cultures were negative. Naso-pharyngeal aspirations were positive 


\begin{tabular}{|c|c|c|c|}
\hline Median & Population A ( $n=35)$ & Population B $(n=127)$ & $P$ \\
\hline Age (months) & 4.55 & 3.51 & 1.00 \\
\hline Weight $(\mathrm{kg})$ & 5.66 & 6.36 & 0.455 \\
\hline $\mathrm{SpO}_{2}$ admission (\%) & 97 & 97 & 0.532 \\
\hline $\mathrm{SpO}_{2}$ minimal $(\%)$ & 93 & 94 & 0.828 \\
\hline Temperature $\left({ }^{\circ} \mathrm{C}\right)$ & 37.9 & 37.5 & 0.004 \\
\hline Heart rate (bpm) & 169 & 163 & 0.331 \\
\hline Respiratory rate (bpm) & 63.5 & 60 & 0.033 \\
\hline $\mathrm{CRP}(\mathrm{mg} / \mathrm{L})$ & 11.9 & 7.7 & 0.091 \\
\hline Neutrophil cells count $(\mathrm{el} / \mu \mathrm{L})$ & 5000 & 4500 & 0.640 \\
\hline
\end{tabular}

bpm: beats per minute; $\mathrm{nb} / \mathrm{m}$ : numbers per minute; el $/ \mu \mathrm{L}$ : elements per microliter. Student's $t$-test with a significance level $<0.05$.

Table 2 : Comparative table of populations with PVA (Pop A) and without PVA inserted in the ER (Pop B). Student's t-test with a significance level $<0.05$.

\begin{tabular}{llll}
\hline Median & $\begin{array}{l}\text { Population A } \\
(n=35)\end{array}$ & $\begin{array}{l}\text { Population B } \\
(n=127)\end{array}$ & $P$ \\
\hline Age (months) & 4.55 & 3.51 & 1.00 \\
Weight (kg) & 5.66 & 6.36 & 0.455 \\
$\mathrm{SpO}$ admission (\%) & 97 & 97 & 0.532 \\
$\mathrm{SpO}_{2}$ minimal (\%) & 93 & 94 & 0.828 \\
Temperature ( $\left.{ }^{\circ} \mathrm{C}\right)$ & 37.9 & 37.5 & 0.004 \\
Heart rate (bpm) & 169 & 163 & 0.331 \\
Respiratory rate (nb/m) & 63.5 & 60 & 0.033 \\
CRP (mg/L) & 11.9 & 7.7 & 0.091 \\
Neutrophil cells count (el/pL) & 5000 & 4500 & 0.640
\end{tabular}

PVA: peripheral vascular access. CRP: C-reactive protein; bpm: beats per minute; nb/m: numbers per minute; el/uL: elements per microliter. Student's $t$-test with a significance level $<0.05$.

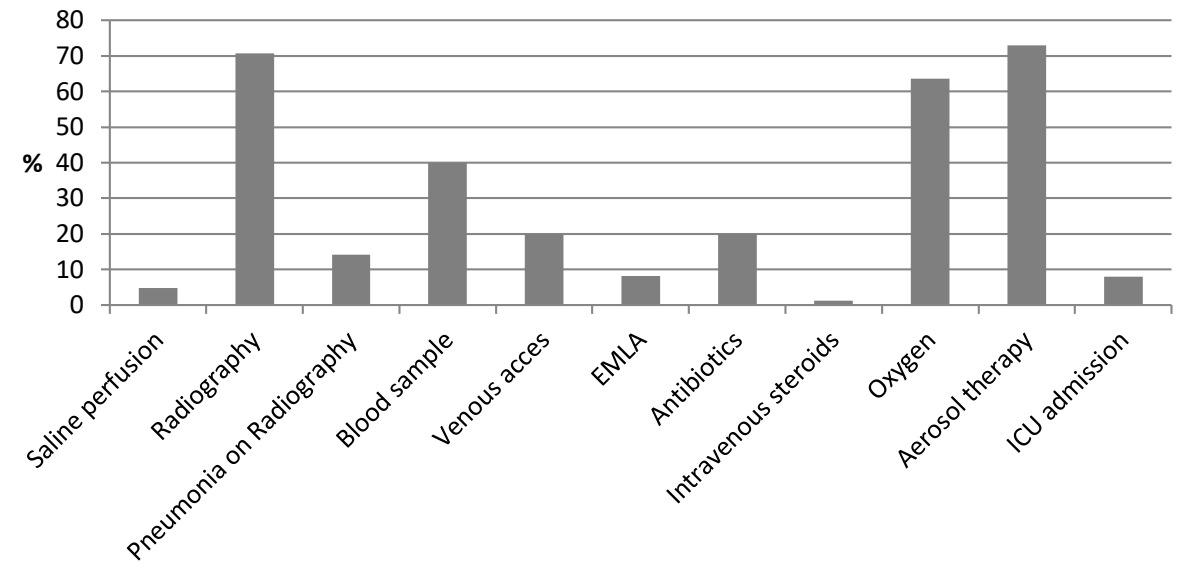

Figure 1: Table initial management in the emergency department (ED). EMLA: eutectic mixture of $25 \mathrm{mg} / \mathrm{g}$ lignocaine plus $25 \mathrm{mg} / \mathrm{g}$ prilocaine anesthesia.

for respiratory viruses. Distribution is shown in Figure 2.

Twenty-five patients received intravenous antibiotic therapy during hospitalization, 5 patients had oral feeding cessation with intravenous hydration. No VA was inserted secondarily during hospital stay on standard wards. Twelve patients were secondarily transferred to the PICU after a mean interval of $3.3 \pm 1.81$ days. Among them, 3 came from pop
A and 9 from B. One of the VA of pop A was used for rapid sequence intubation. VA was inserted in 3 patients of pop B and used only in 1 for rapid sequence intubation. 8 patients were treated by non-invasive ventilation (NIV) (8 in pop B). One patient (NS) received high flow oxygen ( 1 in pop A) and one patient was treated with low flow oxygen. Two patients were treated by invasive ventilation (1 


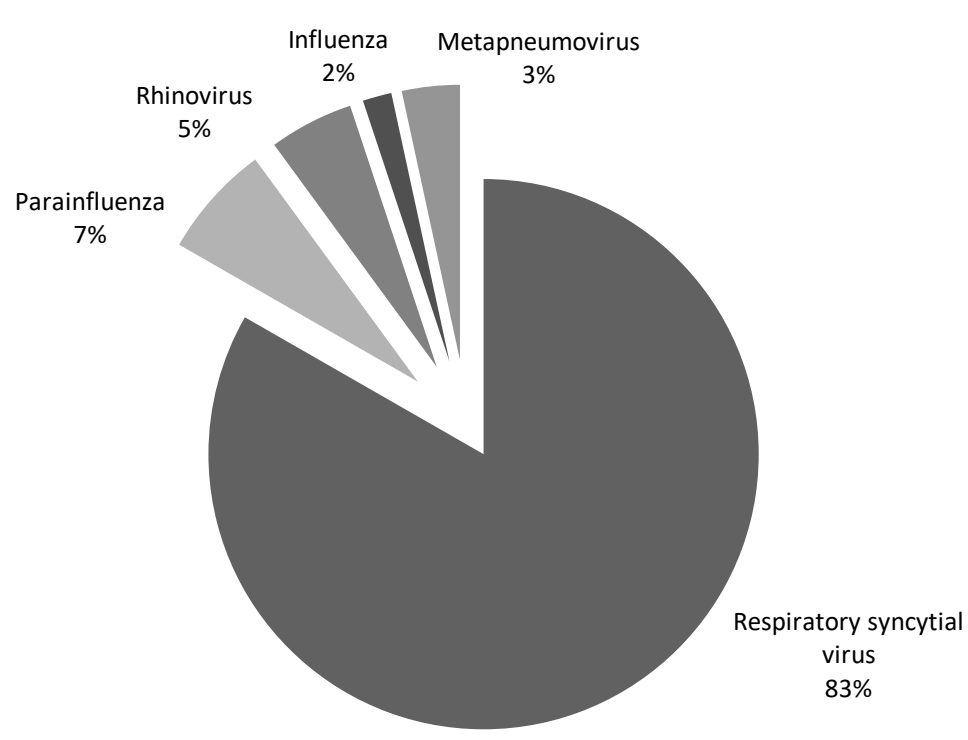

Figure 2: Viral distribution of bronchiolitis

in pop A and 1 in pop B). One of these intubated patients was treated by ECMO. There were no deaths. Mean length of stay was $(9.3 \pm 7.4$ day in pop $A, 7$ and $10 \pm 6.7$ days in $\mathrm{B}, \mathrm{NS})$. No VA was used for adrenaline injection in resuscitation after secondary worsening. No patients were lost during follow-up.

We proceeded to conduct a multivariate analysis with the following as co-factors: age $<6$ months, age $<3$ months, food intake $<60 \%$, temperature $>38^{\circ} \mathrm{C}$, heart rate $>180$ bpm, respiratory rate $>60 / \mathrm{min}, \mathrm{SpO}_{2}<95 \%, \mathrm{SpO}_{2}<$ $90 \%$, Oxygen therapy, positive RSV sampling or suspicion of pneumonia on chest X-ray. We tested these variables on having a catheter placed in the emergency room and on being admitted secondarily to intensive care after hospitalization in a normal ward. We did not find any influence of these variables on the risk factors analyzed (Table 3 and 4).

\section{DISCUSSION}

A single VA inserted in the emergency department was used for rapid sequence intubation after 3 days of hospitalization in a non-intensive ward and after a 1-hour observation with NIV in the ICU. Another patient was intubated with a catheter inserted in the ICU after 5 days of hospitalization and after 24 hours of NIV. We had no patients who required immediate administration of resuscitation drugs following a sudden worsening of their condition. The indication of VA insertion in the ER was not based on the clinical condition as we observed in our multivariate analysis.

In an ICU, compensated respiratory failure requires
Table 3 : Multivariate regression on the risk to receive a venous access in the emergency department.

\begin{tabular}{|c|c|c|c|c|}
\hline \multirow{2}{*}{$\begin{array}{l}\text { Variables } \\
\text { Pneumonia }\end{array}$} & \multirow{2}{*}{$\begin{array}{l}\begin{array}{l}\text { Estimated } \\
\text { OR }\end{array} \\
0.133\end{array}$} & \multicolumn{2}{|c|}{ IC $95 \%$} & \multirow{2}{*}{$\begin{array}{l}P \\
0.27\end{array}$} \\
\hline & & -0.104 & 0.371 & \\
\hline \multicolumn{5}{|l|}{ Age } \\
\hline$<6$ months & -0.064 & -0.394 & 0.263 & 0.69 \\
\hline$<3$ months & 0.046 & -0.244 & 0.334 & 0.75 \\
\hline Food intake $<60 \%$ & 0.041 & -0.189 & 0.272 & 0.72 \\
\hline Temperature $>38^{\circ} \mathrm{C}$ & 0.050 & -0.219 & 0.328 & 0.71 \\
\hline Heart rate $>180 \mathrm{bpm}$ & 0.001 & -0.082 & 0.061 & 0.96 \\
\hline Respiratory rate $>60 / \mathrm{min}$ & 0.077 & -0.159 & 0.316 & 0.52 \\
\hline \multicolumn{5}{|l|}{$\mathrm{SpO}_{2}$} \\
\hline$<95 \%$ & 0.046 & -0.287 & 0.351 & 0.84 \\
\hline$<90 \%$ & 0.037 & -0.303 & 0.387 & 0.83 \\
\hline Oxygen therapy & -0.098 & -0.404 & 0.209 & 0.52 \\
\hline RSV & 0.007 & -0.241 & 0.252 & 0.94 \\
\hline
\end{tabular}

RSV: respiratory syncytial virus. No co-variable is associated with the risk.

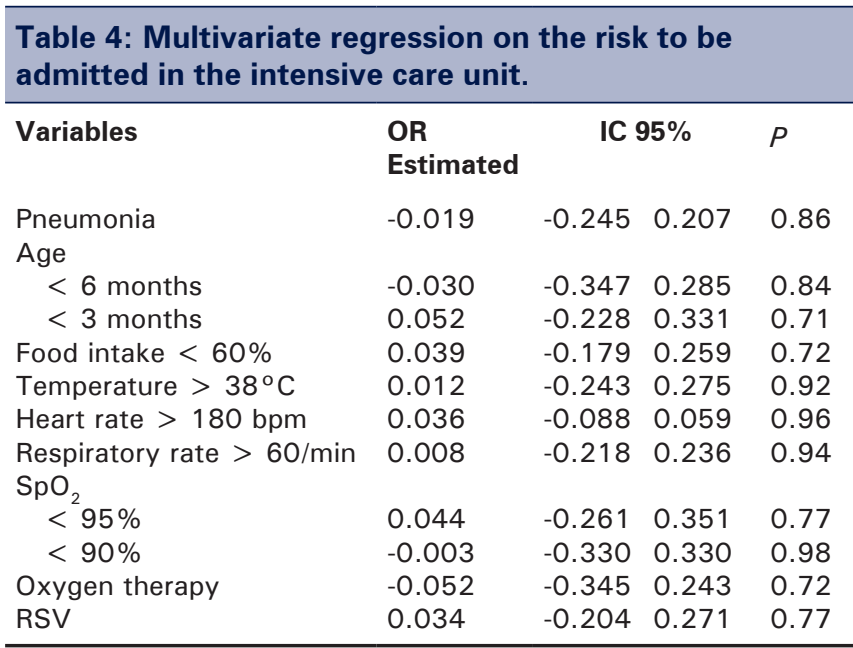

RSV: respiratory syncytial virus. No co-variable is associated with the risk. 
treatment with oxygen, high-flow oxygen therapy or NIV using continuous a positive airway pressure (CPAP) or a bi-level positive airway pressure (BiPAP) type. In our ICU guidelines, food and water intake is then provided by NGT. When the patient deteriorates towards decompensated respiratory failure, mechanical assistance was required and venous access was justified for the administration of a rapid sequence intubation drug. VA was also needed to ensure an effective fluid loading in the event of vasoplegia following the administration of a sedative drug or the significant fall in $\mathrm{PCO}_{2}$ following mechanical ventilation. ${ }^{[7]}$

The administration route of antimicrobial therapy, which could be a potential indication of VA placement, has been studied in two large studies comparing oral versus intravenous. These two studies showed equivalence between oral amoxicillin and intravenous penicillin. ${ }^{[8,9]}$ The use of an antimicrobial therapy per se is also controversial. A Cochrane meta-analysis published in 2014 summarized 7 randomized controlled trials in 824 patients with bronchiolitis under two years of age, comparing the antimicrobial therapy (ampicillin, azithromycin) versus the placebo. No benefit was demonstrated in terms of survival, length of stay, oxygen requirement and readmission rate. ${ }^{[10]} \mathrm{A}$ frequently observed drift is to consider that any patient hospitalized is at risk of worsening cardiorespiratory condition, and therefore, insert a VA upon patient's arrival. Our study is the first to evaluate such a practice and has not shown any benefit in terms of prevention of secondary worsening.

Regarding admission to intensive care, we did not find any predictive factor. Our study was not powerful enough to identify this criteria. In a large multicenter study involving 2,104 patients, Hasegawa et al. showed that low birth weight and polypnea were risk factors for admission to ICU. ${ }^{[11]}$ Similarly, they had a significant difference on oxygen saturation in a monovariate analysis. Polypnea and hypoxia are signs of decompensated respiratory failure, which require high-flow oxygen therapy (high flow oxygen therapy or nasal CPAP) or even invasive ventilation in case of respiratory failure complicated by hypercapnic acidosis.

\section{LIMITATIONS}

This prospective study is strictly observational and was conducted during a single winter. The single center analysis on a small population makes it difficult to draw conclusions, but rather helps to give suggestions. Obviously, only multicenter randomized controlled trials could definitively answer these questions.

\section{CONCLUSION}

In our study, we did not find any situation justifying the preventive insertion of venous access in the ED. Medical treatment may be given orally in most cases. Antimicrobial therapy treatment is not recommended. Management of acute bronchiolitis in ED, apart from decompensated cases of respiratory insufficiency, is handling a respiratory dysfunction, rehydration and nutrition, which in the vast majority do not require any vascular access.

\section{Conflict of interest}

Patrick M. Honore is the Co-Eidtor-in-Chief of the journal. This article was subject to the journal's standard procedures, with peer review handled independently of this editor and his research groups.

\section{REFERENCES}

1. American Academy of Pediatrics Subcommittee on Diagnosis and Management of Bronchiolitis. Diagnosis and of Management Bronchiolitis. Pediatrics 2006; 118: 1774-93.

2. Oakley F, Borland M, Neutze J, Acworth J, Krieser D, Dalziel S, et al. Nasogastric hydration versus intrave $\neg$ nous hydration for infants with bronchiolitis: a randomized trial. The Lancet Respir Med 2013; 1: 113-20.

3. Ralston S, Lieberthal A, Meissner H, Alverson BK, Baley JE, Gadomski AM, et al. Clinical Practice Guideline: The Diagnosis, Management, and Prevention of Bronchiolitis. Pediatrics 2014; 134: e1474-502.

4. Kugelman A, Raibin K, Dabbah H, Chistyakov I, Srugo I, Even L, et al. Intravenous fluids versus gastric-tube feeding in hospitalized infants with viral bronchiolitis: a randomized, prospective pilot study. J Pediatr 2013; 162: 640-2.

5. Macias CG, Mansbach JM, Fisher ES, Riederer M, Piedra PA, Sullivan $\mathrm{AF}$, et al. Variability in inpatient management of children hospitalized with bronchiolitis. Acad Pediatr 2015; 15: 69-76.

6. Babl FE, Sheriff N, Neutze J, Borland M, Oakley E. Bronchiolitis manage $\neg$ ment in pediatric emergency departments in Australia and New Zealand: a PREDICT study. Pediatr Emerg Care 2008; 24: 656-8.

7. Maconochie IK, Bingham R, Eich C, López-Herce J, Rodríguez-Núñez A, Rajka T, et al. European Resuscitation Council Guidelines for Resuscitation 2015: Section 6. Paediatric life support. Resuscitation 2015; 95 : 223-48.

8. Atkinson M, Lakhanpaul, Smyth A, Vyas H, Weston V, Sithole J, et al. Comparison of oral amoxicillin and intravenous benzyl penicillin for community acquired pneumonia in children (PIVOT trial): a multicentre pragmatic randomised controlled equivalence trial. Thorax 2007; 62: 1102-6.

9. Addo-Yobo E, Chisaka N, Hassan M, Hibberd P, Lozano JM, Jeena PM, et al. Oral amoxicillin versus inject $\neg$ able penicillin for severe pneumonia in children aged 3 to 59 months: a randomized multicentre equivalency study. Lancet 2004; 364: 1141-8.

10. Farley R, Spurling G, Eriksson L, Mar CD. Antibiotics for bronchiolitis in children under two years of age. Cochrane Database Syst Rev 2014; (10): CD005189.

11. Hasegawa K, Pate BM, Mansbach JM, Macias CG, Fisher ES, Piedra PA, et al. Risk factors for requiring intensive care among children admitted to ward with bronchiolitis. Acad Pediatr 2015; 15: 77-81.

How to cite this article: Redant S, Nehar-Stern N, Honoré PM, Attou $\mathrm{R}$, Haggenmacher $\mathrm{C}$, et al. Acute bronchiolitis: Why put an IV line? J Transl Intern Med 2021; 9: 185-9. 